\title{
Effect of high- versus low-volume saline administration on acute kidney injury after cardiac surgery
}

\author{
Ju Yong Lim ${ }^{1}$, Pil Je Kang ${ }^{2}$, Sung Ho Jung ${ }^{2}$, Suk Jung Choo ${ }^{2}$, Cheol Hyun Chung ${ }^{2}$, Jae Won Lee ${ }^{2}$, \\ Joon Bum Kim ${ }^{2}$ \\ ${ }^{1}$ Departments of Thoracic and Cardiovascular Surgery, Anam Hospital, University of Korea College of Medicine, Seoul, Republic of Korea; \\ ${ }^{2}$ Department of Thoracic and Cardiovascular Surgery, Asan Medical Center, University of Ulsan College of Medicine, Seoul, Republic of Korea \\ Contributions: (I) Conception and design: JY Lim, PJ Kang; (II) Administrative support: SH Jung, JB Kim; (III) Provision of study materials or \\ patients: SH Jung, SJ Choo, CH Chung, JW Lee; (IV) Collection and assembly of data: JY Lim, PJ Kang; (V) Data analysis and interpretation: JY \\ Lim, JB Kim; (VI) Manuscript writing: All authors; (VII) Final approval of manuscript: All authors. \\ Correspondence to: Joon Bum Kim, MD. Department of Thoracic and Cardiovascular Surgery, Asan Medical Center, University of Ulsan College of \\ Medicine, 88, Olympic-ro 43-gil, Songpa-gu, Seoul 05505, Republic of Korea. Email: jbkim1975@amc.seoul.kr.
}

\begin{abstract}
Background: Fluid resuscitation is critical to perioperative maintenance of adequate preload and cardiac output after cardiac surgery. Liberal use of saline, however, is reportedly associated with an increased risk of acute kidney injury (AKI) in critically ill patients. This study examined the effects of high-versus low-volume saline administration on AKI after cardiac surgery.

Methods: In this retrospective study, we evaluated 1,740 consecutive patients who underwent cardiac surgery over a 2 -year period. The patients were divided into high-volume saline ( $\mathrm{n}=328,18.8 \%)$ and lowvolume saline $(n=1,412,81.2 \%)$ groups based on the amount of saline ( $>1$ or $\leq 1 \mathrm{~L}$, respectively) administered during the first 48 postoperative hours.

Results: AKI, the primary outcome, was defined according to the Risk, Injury, Failure, Loss, End Stage classification. There were no significant differences in the incidence of AKI $(\mathrm{P}=0.46)$, new renal replacement therapy (RRT) $(\mathrm{P}=0.39)$, and early mortality $(\mathrm{P}=0.52)$ between the 2 groups. Adjustment of baseline characteristics using propensity score matching showed that high-volume of saline administration was not significantly associated with an increased risk of AKI (OR, 1.22; 95\% CI, 0.77-1.93; P=0.38), new RRT (OR, 1.25; 95\% CI, 0.68-2.28; $\mathrm{P}=0.45$ ), or early mortality (HR, 0.98; 95\% CI, 0.48-2.02; P=0.97). These results were validated by further adjustments for significant covariates.
\end{abstract}

Conclusions: High-volume administration of saline in the period following cardiac surgery was not associated with a significant increase in the risk of AKI.

Keywords: Acute kidney injury (AKI); crystalloid solution; cardiac surgery; cardiopulmonary bypass (CPB)

Submitted Jul 12, 2018. Accepted for publication Sep 25, 2018.

doi: $10.21037 /$ jtd.2018.10.113

View this article at: http://dx.doi.org/10.21037/jtd.2018.10.113

\section{Introduction}

Fluid resuscitation to increase intravascular volume is a common and crucial part of patient management in the intensive care unit (ICU) and surgical ICU (1). Fluid management is especially critical after cardiac surgery in order to maintain adequate preload and cardiac output. The literature recommends use of crystalloid solution for this purpose (2,3), with normal saline being most commonly used $(4,5)$. Recent studies, however, have raised concern that excessive use of normal saline may induce hyperchloremic acidosis and subsequent acute kidney injury (AKI) (6-10). As an alternative to normal saline, balanced solutions have become widely used in order to better preserve renal function in ICU settings. For instance, the British Consensus Guidelines on intravenous fluid therapy for adult surgical patients recommend that balanced salt solutions 


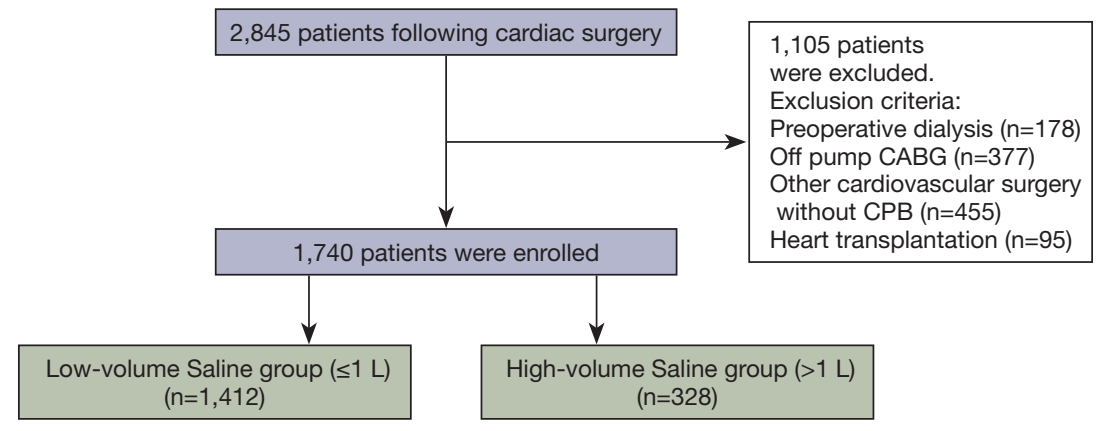

Figure 1 Study flowchart. CABG, coronary artery bypass graft; CPB, cardiopulmonary bypass.

should replace $0.9 \%$ saline when crystalloid resuscitation is indicated, to reduce the risk of hyperchloremic acidosis (11). However, there is insufficient evidence regarding the applicability of intravenous fluid therapy in cardiac surgical patients, who may be particularly vulnerable to AKI following cardiopulmonary bypass (CPB) (12). Therefore, we compared the effects of high- and low-volume saline administration on postoperative renal function in patients who underwent cardiac surgery with $\mathrm{CPB}$.

\section{Methods}

\section{Study population}

From January 2014 to December 2015, 2,845 consecutive adult patients were admitted to the ICU following cardiac surgery at Asan Medical Center in Seoul, Korea. Of these, we excluded patients who underwent cardiac surgery without CPB support, those who underwent heart transplantation, and those who preoperatively received renal replacement therapy (RRT). Finally, 1,740 consecutive patients were enrolled, and their medical records were retrospectively reviewed. The study flow chart is shown in Figure 1. This study was approved and informed consent was waived by the Institutional Review Board (IRB) of our institution due to its retrospective nature (IRB number: 2016-0481).

\section{Intraoperative fluid management}

In the operating room, balanced buffered solution (Plasma Solution A, chloride concentration $98 \mathrm{mmol} / \mathrm{L}$; CJ Healthcare, Seoul, Korea) was used as the priming solution for all CPB and for any additional volume requirements during $\mathrm{CPB}$. Mean blood pressure was maintained at around $60 \mathrm{mmHg}$ during $\mathrm{CPB}$. Upon $\mathrm{CPB}$ cessation, fluid was administered to obtain proper hemodynamic stability based on volume status on transesophageal echocardiography. After discontinuation of $\mathrm{CPB}$, a renal protective fluid strategy consisting of balanced crystalloid solution and a limited amount of hydroxyethyl starch (HES) (Hextend or Volulyte, CJ Healthcare) (13) was performed by an anesthesiologist. Perioperative blood product transfusion and fluid administration were performed according to the protocol of our institution.

\section{Postoperative fluid management}

The fluid management protocol at our ICU included initial fluid resuscitation using up to $1 \mathrm{~L}$ of crystalloid solution. If additional volume was required, additional crystalloid solution was used, provided that the patient was well-oxygenated. Two kinds of crystalloid solution were used: a chloride-rich solution $(0.9 \%$ saline, chloride concentration $150 \mathrm{mmol} / \mathrm{L}$; JW Pharmaceutical, Seoul, Korea) and a balanced buffered solution (Plasma Solution A; CJ HealthCare). The choice of the crystalloid solution was at the physician's discretion. If crystalloid infusion was not effective as a volume expander, $6 \%$ HES solutions [Hextend 670/0.75, JW Pharmaceutical, or Volulyte (130/0.4), Fresenius Kabi, Bad Homburg, Germany] were used up to $20 \mathrm{~mL} / \mathrm{kg}$ as a second line of fluid according to the patient's renal function and coagulation. As a routine practice, albumin was not used as a volume expander in the immediate postoperative period. As the primary interest of our study was to evaluate the effect of high-volume saline administration on AKI following cardiac surgery, the patients were divided into 2 groups, i.e.,- -high-volume saline ( $>1 \mathrm{~L})$ and low-volume saline $(\leq 1 \mathrm{~L})$, according to the amount of saline administered during the first 48 hours after surgery regardless of fluid management strategy. 


\section{Outcomes}

The primary outcome was the incidence of postoperative AKI, as defined by Kidney Disease: Improving Global Outcomes (KDIGO) (stage 1: increase in serum creatinine of 1.5-1.9 times the baseline value, stage 2 : increase in serum creatinine of 2.0-2.9 times baseline, and stage 3: increase in serum creatinine of $\geq 3.0$ times baseline or initiation of new RRT), and the risk, injury, and failure stages of the Risk, Injury, Failure, Loss, End Stage classification (14), based on the peak serum creatinine level within 2 postoperative days. Secondary outcomes were the need for RRT and in-hospital mortality. Baseline creatinine was the most recent available preoperative level. The creatinine level was measured daily during the first 2 postoperative days.

\section{Statistical analysis}

Statistical analyses were performed using SAS version 9.3 (SAS Institute, Cary, NC, USA). Data are expressed as mean \pm SD for continuous variables, and as numbers and percentages for categorical variables. Pre- and postoperative measurements were compared using Student's $t$-test or the Mann-Whitney U-test. The chi-square test or Fisher's exact test was used to compare categorical variables and to assess the statistical significance of differences between the 2 groups. A P value of $\leq 0.05$ was considered statistically significant in all comparisons. Univariable and multivariable analyses were performed for the entire patient cohort by using logistic regression for AKI and Cox proportional hazards model for in-hospital mortality to identify the associations with early adverse outcomes. Variables were included in multivariable analysis if their univariable significance was $<0.1$. Backward elimination method was used for variable selection.

To reduce the effect of treatment-selection bias and potential confounders in this observational study, we performed rigorous adjustment for significant differences in the baseline patient characteristics using propensity score matching $(15,16)$. A propensity score was generated for each patient from a multivariable logistic regression model based on 15 preoperative characteristic variables in Table 1 [age, sex, diabetes mellitus, hypertension, cerebrovascular accident, EuroSCORE (European System for Cardiac Operative Risk Evaluation), left ventricular ejection fraction, medications, and laboratory findings] as independent variables, with saline group (saline $>1$ versus $\leq 1 \mathrm{~L})$ as a binary dependent variable. At most, $<3 \%$ of values were missing. To create the propensity score, a single imputation using the Monte Carlo method was used to fill out incomplete baseline variables with the assumption that data were missing at random. The discrimination and calibration abilities of each propensity score model were assessed using $\mathrm{C}$ and Hosmer-Lemeshow statistics. The low-volume saline group was then matched with the high-volume saline group in a 1:4 (maximum) ratio using a greedy matching algorithm. After matching, we compared the baseline covariates between the 2 groups as the standardized difference of means. In the propensity score-matched cohort, the risk of each outcome was compared using a logistic regression model with generalized estimating equations for AKI, KDIGO, and new RRT, and Cox proportional hazard models with robust standard errors and a sandwich covariance matrix estimation for inhospital mortality, which accounted for the clustering of matched pairs. Clinically significant perioperative covariates that were not considered in propensity score matching were also adjusted in this analysis.

\section{Results}

Of the 1,740 patients, $328(18.8 \%)$ were included in the high-volume saline group and $1,412(81.2 \%)$ in the low-volume saline group. The mean amounts of saline administered were $2.47 \pm 1.51$ and $0.54 \pm 0.32 \mathrm{~L}$ in the highand low-volume saline group, respectively. The baseline patient characteristics are summarized in Table 1. Patients in the high-volume saline group had significantly higher baseline creatinine levels $(\mathrm{P}=0.03)$. More fresh frozen plasma (FFP) was perioperatively transfused in this group $(\mathrm{P}=0.006)$, and hyperchloremia was also more frequently observed $(\mathrm{P}=0.06)$. The amount of balanced buffered solution and HES used were similar between the 2 groups. Postoperative findings are summarized in Table 2.

\section{Risk-adjusted outcomes}

To reduce confounding factors in the assessment of the effect of saline after cardiac surgery, we performed propensity score-matched analysis. A total of 978 patients in the low-volume saline group were matched with 291 patients in the high-volume saline group (HosmerLemeshow statistic $\mathrm{P}=0.69, \mathrm{C}$-statistic value $=0.68$ ) (Table 3). The propensity-matched analysis did not show any significant differences in the outcomes between the 
Table 1 Preoperative patient characteristics

\begin{tabular}{|c|c|c|c|c|}
\hline Preoperative variables & Low-volume saline $(n=1,412)$ & High-volume saline $(n=328)$ & $P$ value & Standardized difference of mean \\
\hline Sex (male) & $758(53.6)$ & $190(57.9)$ & 0.16 & 0.08 \\
\hline DM & $218(15.4)$ & $61(18.6)$ & 0.15 & 0.08 \\
\hline HTN & $573(40.6)$ & $141(43.0)$ & 0.40 & 0.05 \\
\hline EuroSCORE & $2.9 \pm 4.8$ & $2.6 \pm 4.9$ & 0.04 & 0.05 \\
\hline LVEF, \% & $58.7 \pm 10.3$ & $58.0 \pm 10.9$ & 0.33 & 0.06 \\
\hline \multicolumn{5}{|l|}{ Medication } \\
\hline ARB & $470(33.3)$ & $108(32.9)$ & 0.90 & 0.01 \\
\hline Statins & $462(32.7)$ & $111(33.8)$ & 0.69 & 0.02 \\
\hline \multicolumn{5}{|l|}{ Laboratory } \\
\hline Hemoglobin, g/dL & $12.7 \pm 1.9$ & $12.7 \pm 2.1$ & 0.77 & 0.02 \\
\hline Creatinine, mg/dL & $0.87(0.72,1.05)$ & $0.9(0.75,1.09)$ & 0.03 & 0.18 \\
\hline $\mathrm{GFR}, \mathrm{mL} / \mathrm{min} / 1.73 \mathrm{~m}^{2}$ & $76.8 \pm 29.0$ & $74.4 \pm 31.5$ & 0.19 & 0.07 \\
\hline BUN, mg/dL & $17.9 \pm 9.1$ & $18.5 \pm 9.5$ & 0.55 & 0.06 \\
\hline Total bilirubin, mg/dL & $0.5(0.4,0.8)$ & $0.5(0.4,0.8)$ & 0.24 & 0.05 \\
\hline Albumin, $\mathrm{g} / \mathrm{dL}$ & $3.65 \pm 0.5$ & $3.6 \pm 0.5$ & 0.59 & 0.03 \\
\hline
\end{tabular}

Results are presented as mean \pm SD, number (percentage) or median (IQR). DM, diabetes mellitus; HTN, hypertension; CVA, cerebrovascular accident; EuroSCORE, European System for Cardiac Operative Risk Evaluation; LVEF, left ventricular ejection fraction; ARB, angiotensin receptor blockers; GFR, glomerular filtration rate; BUN, blood urea nitrogen.

Table 2 Perioperative findings

\begin{tabular}{|c|c|c|c|c|}
\hline Perioperative variables & Low-volume saline $(n=1,412)$ & High-volume saline $(n=328)$ & $P$ value & Standardized difference of mean \\
\hline Operation & & & 0.34 & 0.13 \\
\hline Valve & $912(64.6)$ & $201(61.3)$ & & \\
\hline Valve + CABG & $50(3.5)$ & $15(4.6)$ & & \\
\hline Aorta & $200(14.2)$ & $53(16.2)$ & & \\
\hline Others & $163(11.5)$ & $32(9.8)$ & & \\
\hline Postop. ECMO & $42(3.0)$ & $12(3.7)$ & 0.52 & 0.04 \\
\hline
\end{tabular}

Table 2 (continued) 
Table 2 (continued)

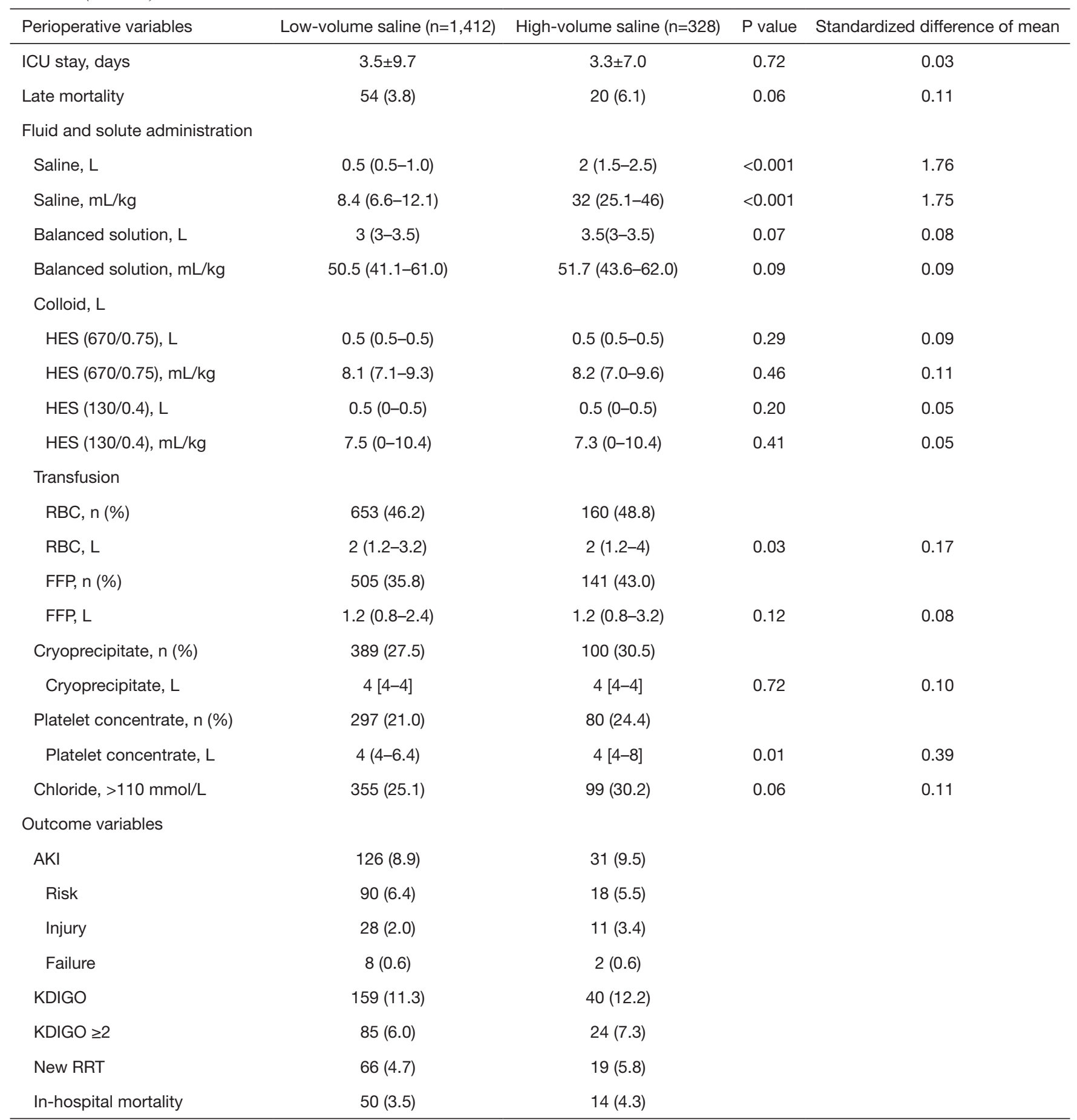

Results are presented as mean $\pm \mathrm{SD}$, number (percentage) or median (IQR). CPB, cardiopulmonary bypass; ACC, aortic cross-clamp; CABG, coronary artery bypass graft; ECMO, extracorporeal membrane oxygenation; RBC, red blood cells; FFP, fresh frozen plasma; HES, hydroxyethyl starch; AKI, acute kidney injury; KDIGO, Kidney Disease: Improving Global Outcomes; RRT, renal replacement therapy; ICU, intensive care unit. 
Table 3 Comparison of propensity score-matched patients

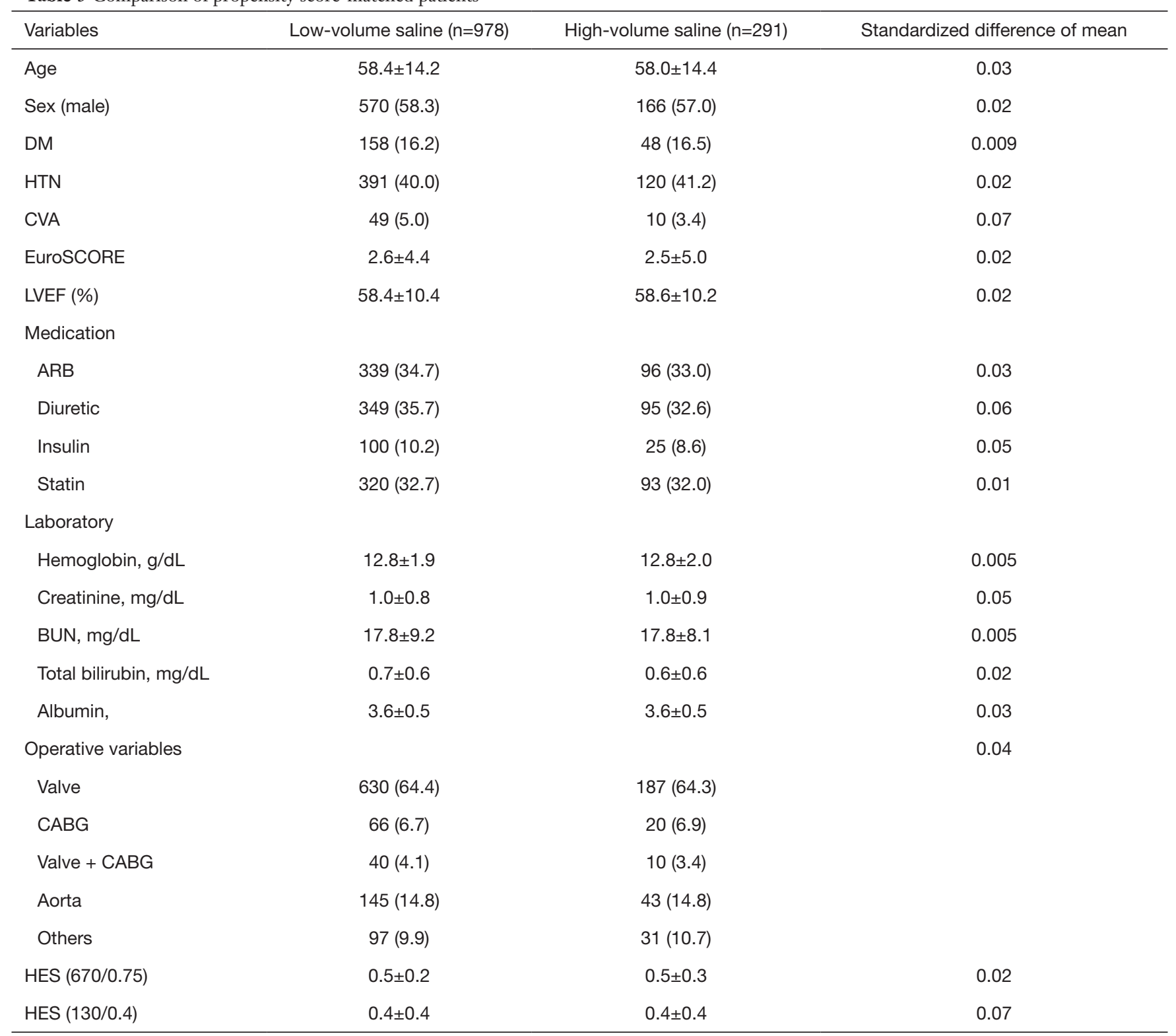

Results are presented as mean \pm SD or number (percentage). DM, diabetes mellitus; HTN, hypertension; CVA, cerebrovascular accident; EuroSCORE, European System for Cardiac Operative Risk Evaluation; LVEF, left ventricular ejection fraction; ARB, angiotensin receptor blocker; BUN, blood urea nitrogen; CABG, coronary artery bypass graft; HES, hydroxyethyl starch.

2 groups in terms of AKI incidence (OR, 1.22; 95\% CI, 0.77-1.93; $\mathrm{P}=0.38)$, need for RRT (OR, 1.25; 95\% CI, $0.68-2.28 ; \mathrm{P}=0.45$ ), and in-hospital mortality (HR, 0.98; 95\% CI, 0.48-2.02; $\mathrm{P}=0.97$ ).

In this propensity score-matched dataset, adjustment was performed for 5 clinically significant covariates, namely, CPB time, use of FFP, cryoprecipitate transfusion, postoperative extracorporeal membrane oxygenation (ECMO), and postoperative bleeding. This analysis also showed that highvolume saline administration did not significantly affect postoperative kidney function or mortality. The analysis of each outcome is summarized in Table 4.

\section{Sensitivity analysis}

In our study, the patients were divided into 2 groups 
Table 4 Analysis of outcomes

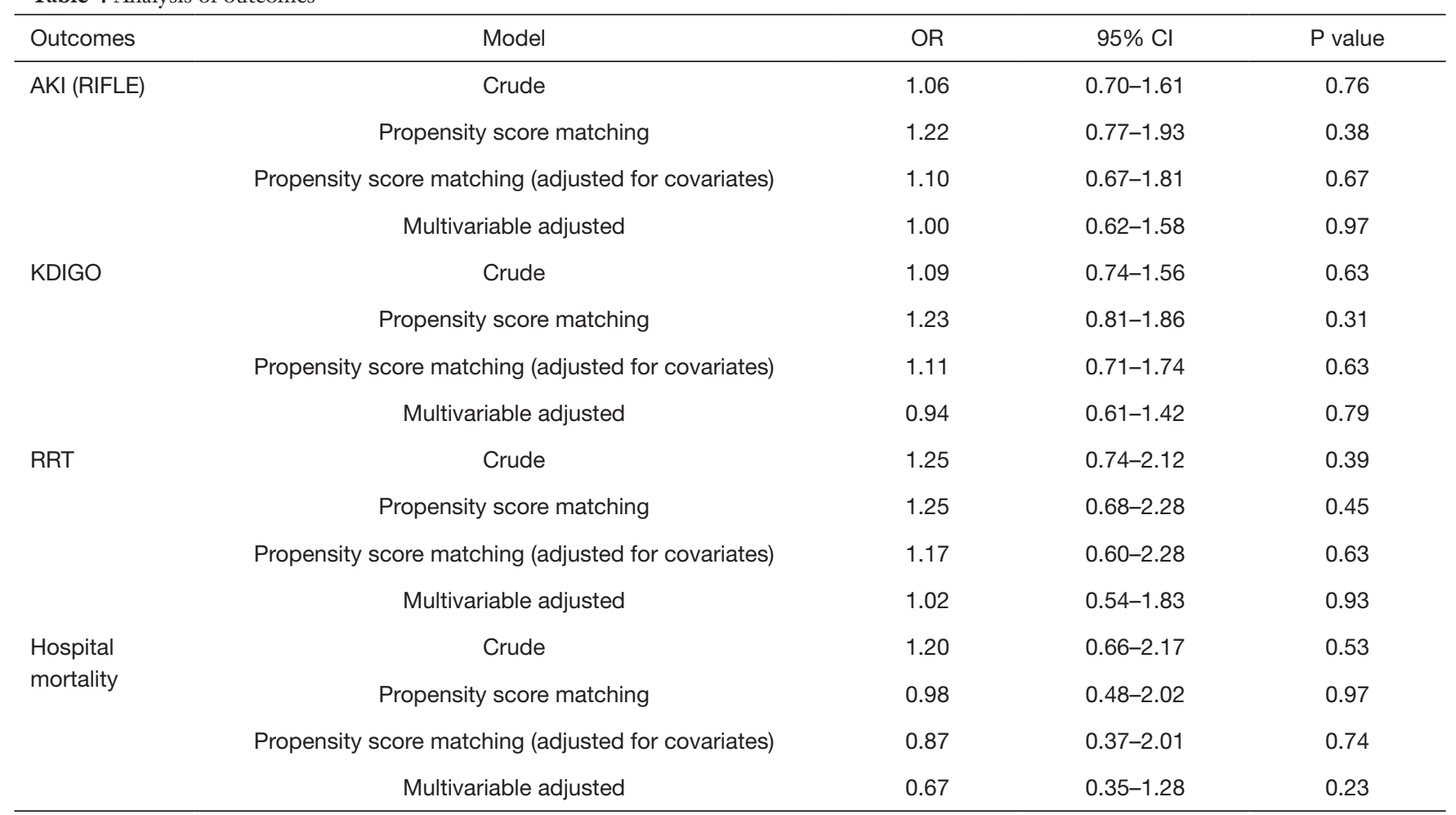

Covariates: cardiopulmonary bypass time, use of fresh frozen plasma, cryoprecipitate transfusion, postoperative extracorporeal membrane oxygenation, and postoperative bleeding. AKI, acute kidney injury; RIFLE, Risk, Injury, Failure, Loss, End Stage; KDIGO, Kidney Disease: Improving Global Outcomes; RRT, renal replacement therapy.

Table 5 Sensitivity analysis

\begin{tabular}{lcccc}
\hline Outcomes & Variables & OR & $95 \% \mathrm{Cl}$ & P value \\
\hline AKI & Total amount of saline & 1.08 & $0.88-1.88$ & 0.41 \\
(RIFLE) & Saline $>1$ L & 1.49 & $0.87-2.57$ & 0.14 \\
& Saline $>2 ~ L$ & 1.70 & $0.71-4.06$ & 0.22 \\
\multirow{2}{*}{ KDIGO } & Total amount of saline & 0.98 & $0.77-1.26$ & 0.92 \\
& Saline $>1$ L & 1.49 & $0.87-2.57$ & 0.14 \\
& Saline $>2$ L & 1.07 & $0.38-3.02$ & 0.88 \\
\hline
\end{tabular}

AKI, acute kidney injury; RIFLE, Risk, Injury, Failure, Loss, End Stage; KDIGO, Kidney Disease: Improving Global Outcomes.

according to the amount of saline administered during the first 48 hours after surgery and the incidence of postoperative AKI was defined based on the peak serum creatinine level within 2 postoperative days. To overcome the duplication of the duration of saline infused and the peak creatinine obtained for defining AKI, additional validity analyses were done. We evaluated the association between the total amount of saline administered on POD\#0 only and we defined AKI according to the peak creatinine level on POD\#2. These analyses showed consistent results with our initial data as summarized in Table 5 .

\section{Determinants of AKI and mortality}

In univariable analysis, the total amount of saline administered did not significantly increase the risk of AKI (OR, 1.06; 95\% CI, 0.70-1.61; $\mathrm{P}=0.76$ ), need for RRT (OR, 1.25 ; 95\% CI, 0.74-2.12; $\mathrm{P}=0.39)$, and in-hospital mortality (HR, 1.20; 95\% CI, 0.66-2.17; $\mathrm{P}=0.53$ ). Multivariable analysis also showed that the total amount of saline administered was not associated with a significant increase in the risk of AKI (OR, 1.00; 95\% CI, 0.62-1.58; $\mathrm{P}=0.97)$, need for RRT (OR, 1.02; 95\% CI, 0.54-1.83; P=0.93), and in-hospital mortality (HR, 0.67; 95\% CI, 0.35-1.28; $\mathrm{P}=0.23)$. Age, $\mathrm{CPB}$ time, FFP transfusion, and postoperative ECMO support were identified as risk factors for AKI, need for RRT, and in-hospital mortality (Tables S1-S3). 


\section{Discussion}

We compared the impact of high- and low-volume saline administration on kidney function after cardiac surgery using $\mathrm{CPB}$, and found that high-volume administration was not significantly associated with an increased risk of postoperative AKI, need for RRT, or in-hospital mortality. These findings remained unchanged after adjustment for baseline characteristics and propensity score matching. Our finding is in line with that of a randomized controlled trial (17) that compared the effect of a buffered crystalloid solution and saline on AKI in critically ill patients, and demonstrated that the incidence of AKI and need for new RRT were similar for both solutions.

Some reports on postoperative fluid management strategy would seem to disagree with our results. A prospective pilot study (18) argued that a chloriderestricted strategy was associated with a significant decrease in the incidence of AKI as well as the need for RRT in critically ill patients. However, this trial was carried out on a relatively heterogeneous population, of which only $40-50 \%$ had cardiovascular disease or were in post-cardiac surgical settings. Another recent study showed that the use of balanced crystalloid solution for fluid resuscitation was associated with lower postoperative mortality and renal failure in patients undergoing abdominal surgery (19). Importantly, the patients in that study also differed from our patients, who had concurrent cardiac disease and possible kidney dysfunction. One retrospective observational study that focused on patients undergoing off-pump coronary artery bypass surgery demonstrated the efficacy of a perioperative renal protective fluid management strategy consisting of balanced solution instead of chloride-rich saline (13). However, the subjects included in that study may not be representative of all patients undergoing cardiac surgery with CPB.

The majority of valvular, coronary, and aortic surgeries are performed with $\mathrm{CPB}$, which carries the risk of multiorgan and kidney injury. The pathophysiology of kidney injury associated with CPB has been attributed to cellular ischemia and consequent injury to tubular epithelium and vascular endothelium in the kidney (20-23). The mean arterial blood pressure during cardiac surgery is often at or below the lower limits of autoregulation. In addition, many patients undergoing cardiac surgery have impaired autoregulation due to preexisting comorbidities such as advanced age, atherosclerosis, or chronic hypertension; these patients may also be receiving drugs that impair kidney autoregulation (e.g., angiotensin receptor blockers) (20).

Postoperative administration of chloride-rich fluid has been examined in vulnerable patients, and some physicians preferred using buffered crystalloid solution to protect renal function. However, in our study, the high-volume saline group did not show significantly worse outcomes in terms of kidney function and survival than the low-volume saline group. Of course, as shown in Table 2, the 2 groups received similar amounts of balanced solution and colloid. The only difference was the total amount of saline. Therefore, patients in the high-volume saline group received more total fluid than the low-volume saline group. This implies that the beneficial effect of hydration with high-volume infusion of saline may outweigh the potential adverse effect of hyperchloremic metabolic acidosis that may result from saline infusion. A recently published randomized controlled trial on the effect of restricting chloride perioperatively also concluded that liberal chloride loading perioperatively was not associated with AKI or new RRT after cardiac surgery (24), which is concordant with our findings. The risk factor analysis in our study identified age, type of operation, $\mathrm{CPB}$ time, postoperative ECMO, and transfusion as risk factors, similar to the findings in a previous report (12). This suggests that liberal postoperative saline fluid management has limited effect on kidney function, while preoperative and operative CPB factors act as strong determinants of kidney injury in this subset of patients. The types of fluid administered after surgery do not seem to significantly affect the postoperative course. In addition, the mean duration of ICU stay after cardiac surgery was 2 days, which is a relatively short time, and hyperchloremia caused by high-volume saline administration over a short duration such as 2 days is unlikely to be associated with an increased risk of AKI and need for RRT.

There are several limitations to our study. First, this was a retrospective, observational study and not a randomized controlled trial. Comparison between the 2 groups may be biased by potential confounding factors, even though we performed propensity score matching and multivariable adjustment analysis to avoid potential bias. Second, the amount of saline administered in the high-volume saline group was $2.47 \mathrm{~L}$ on average, because colloids or blood products were also used to expand the intravascular volume if crystalloid alone was not effective for volume resuscitation. Thus, the total amount of saline may not be enough to affect kidney function in combination with other preoperative and intraoperative factors. 


\section{Conclusions}

High-volume saline administration $(>1 \mathrm{~L})$ after cardiac surgery with $\mathrm{CPB}$ was not significantly associated with an increased risk of AKI, need for RRT, or in-hospital mortality. A further randomized controlled study on the liberal use of saline and its safety in patients undergoing cardiac surgery is necessary for a more decisive recommendation.

\section{Acknowledgements}

This study was supported by a grant from the Asan Institute for Life Sciences and Corporate Relations of Asan Medical Center, Seoul, Korea.

\section{Footnote}

Conflicts of Interest: The authors have no conflicts of interest to declare.

Ethical Statement: This study was approved and informed consent was waived by the Institutional Review Board (IRB) of our institution due to its retrospective nature (IRB number: 2016-0481).

\section{References}

1. Myburgh JA, Mythen MG. Resuscitation fluids. N Engl J Med 2013;369:1243-51.

2. Perel P, Roberts I. Colloids versus crystalloids for fluid resuscitation in critically ill patients. Cochrane Database Syst Rev 2007:CD000567.

3. Virgilio RW, Rice CL, Smith DE, et al. Crystalloid vs. colloid resuscitation: is one better? A randomized clinical study. Surgery 1979;85:129-39.

4. Awad S, Allison SP, Lobo DN. The history of $0.9 \%$ saline. Clin Nutr 2008;27:179-88.

5. Finfer S, Liu B, Taylor C, et al. Resuscitation fluid use in critically ill adults: an international cross-sectional study in 391 intensive care units. Crit Care 2010;14:R185.

6. Hansen PB, Jensen BL, Skott O. Chloride regulates afferent arteriolar contraction in response to depolarization. Hypertension 1998;32:1066-70.

7. Lobo DN, Stanga Z, Aloysius MM, et al. Effect of volume loading with 1 liter intravenous infusions of $0.9 \%$ saline, $4 \%$ succinylated gelatine (Gelofusine) and $6 \%$ hydroxyethyl starch (Voluven) on blood volume and endocrine responses: a randomized, three-way crossover study in healthy volunteers. Crit Care Med 2010;38:464-70.

8. McCluskey SA, Karkouti K, Wijeysundera D, et al. Hyperchloremia after noncardiac surgery is independently associated with increased morbidity and mortality: a propensity-matched cohort study. Anesth Analg 2013;117:412-21.

9. Reid F, Lobo DN, Williams RN, et al. (Ab)normal saline and physiological Hartmann's solution: a randomized double-blind crossover study. Clin Sci (Lond) 2003;104:17-24.

10. Wilcox CS. Regulation of renal blood flow by plasma chloride. J Clin Invest 1983;71:726-35.

11. Soni N. British consensus guidelines on intravenous fluid therapy for adult surgical patients (GIFTASUP): Cassandra's view. Anaesthesia 2009;64:235-8.

12. Karkouti K, Wijeysundera DN, Yau TM, et al. Acute kidney injury after cardiac surgery: focus on modifiable risk factors. Circulation 2009;119:495-502.

13. Kim JY, Joung KW, Kim KM, et al. Relationship between a perioperative intravenous fluid administration strategy and acute kidney injury following off-pump coronary artery bypass surgery: an observational study. Crit Care 2015;19:350.

14. Bellomo R, Ronco C, Kellum JA, et al. Acute renal failure definition, outcome measures, animal models, fluid therapy and information technology needs: the Second International Consensus Conference of the Acute Dialysis Quality Initiative (ADQI) Group. Crit Care 2004;8:R204-12.

15. D'Agostino RB, Jr. Propensity score methods for bias reduction in the comparison of a treatment to a nonrandomized control group. Stat Med 1998;17:2265-81.

16. Rosenbaum PR, Rubin DB. The central role of the propensity score in observational studies for causal effects. Biometrika 1983;70:41-55.

17. Young P, Bailey M, Beasley R, et al. Effect of a buffered crystalloid solution vs saline on acute kidney injury among patients in the intensive care unit: the SPLIT randomized clinical trial. JAMA 2015;314:1701-10.

18. Yunos NM, Bellomo R, Hegarty C, et al. Association between a chloride-liberal vs chloride-restrictive intravenous fluid administration strategy and kidney injury in critically ill adults. JAMA 2012;308:1566-72.

19. Shaw AD, Bagshaw SM, Goldstein SL, et al. Major complications, mortality, and resource utilization after open abdominal surgery: $0.9 \%$ saline compared to PlasmaLyte. Ann Surg 2012;255:821-9.

20. Abuelo JG. Normotensive ischemic acute renal failure. $\mathrm{N}$ Engl J Med 2007;357:797-805. 
21. Krian A. Incidence, prevention, and treatment of acute renal failure following cardiopulmonary bypass. Int Anesthesiol Clin 1976;14:87-101.

22. Sutton TA, Fisher CJ, Molitoris BA. Microvascular endothelial injury and dysfunction during ischemic acute renal failure. Kidney Int 2002;62:1539-49.

23. Yeboah ED, Petrie A, Pead JL. Acute renal failure and

Cite this article as: Lim JY, Kang PJ, Jung SH, Choo SJ, Chung CH, Lee JW, Kim JB. Effect of high- versus low-volume saline administration on acute kidney injury after cardiac surgery. J Thorac Dis 2018;10(12):6753-6762. doi: 10.21037/ jtd.2018.10.113 open heart surgery. Br Med J 1972;1:415-8.

24. McIlroy D, Murphy D, Kasza J, et al. Effects of restricting perioperative use of intravenous chloride on kidney injury in patients undergoing cardiac surgery: the LICRA pragmatic controlled clinical trial. Intensive Care Med 2017;43:795-806. 
Table S1 Analysis of risk factors for acute kidney injury (risk, injury, and failure stage as defined by the RIFLE criteria)

\begin{tabular}{|c|c|c|c|c|c|c|}
\hline \multirow{2}{*}{ Variables } & \multicolumn{3}{|c|}{ Univariate analysis } & \multicolumn{3}{|c|}{ Multivariable analysis } \\
\hline & OR & $95 \% \mathrm{Cl}$ & $P$ value & OR & $95 \% \mathrm{Cl}$ & $P$ value \\
\hline \multicolumn{7}{|l|}{ Preoperative variables } \\
\hline Sex (male) & 1.17 & $0.84-1.62$ & 0.35 & & & \\
\hline Age & 1.03 & $1.01-1.04$ & $<0.001$ & 1.02 & $1.01-1.04$ & 0.004 \\
\hline Type of operation & & & 0.00 & & & \\
\hline CABG & 0.96 & $0.47-1.97$ & 0.92 & & & \\
\hline Valve + CABG & 1.80 & $0.86-3.77$ & 0.12 & & & \\
\hline Aorta & 2.05 & $1.37-3.06$ & $<0.001$ & & & \\
\hline Others & 0.54 & $0.27-1.10$ & 0.09 & & & \\
\hline DM & 1.71 & $1.15-2.53$ & 0.01 & & & \\
\hline HTN & 1.28 & $0.92-1.77$ & 0.15 & & & \\
\hline CVA & 1.16 & $0.57-2.35$ & 0.69 & & & \\
\hline EuroSCORE & 1.05 & $1.03-1.08$ & $<0.001$ & & & \\
\hline LVEF & 0.99 & $0.97-1.00$ & 0.12 & & & \\
\hline \multicolumn{7}{|l|}{ Medication } \\
\hline ARB & 1.23 & $0.88-1.73$ & 0.22 & & & \\
\hline Diuretic & 1.61 & $1.16-2.24$ & 0.00 & & & \\
\hline Hypoglycemic agent & 2.09 & $1.36-3.21$ & 0.00 & & & \\
\hline ACE inhibitor & 1.71 & $1.07-2.73$ & 0.02 & & & \\
\hline Insulin & 2.32 & $1.49-3.59$ & $<0.001$ & 2.40 & $1.43-3.94$ & 0.001 \\
\hline Statin & 1.41 & $1.01-1.97$ & 0.05 & & & \\
\hline Antiplatelet & 1.71 & $1.23-2.39$ & 0.00 & & & \\
\hline Hemoglobin & 0.82 & $0.75-0.89$ & $<0.001$ & & & \\
\hline Creatinine & 0.73 & $0.50-1.05$ & 0.09 & & & \\
\hline GFR & 0.99 & $0.98-1.00$ & 0.08 & & & \\
\hline Total bilirubin & 1.27 & $1.06-1.52$ & 0.01 & & & \\
\hline Albumin & 0.44 & $0.33-0.58$ & $<0.001$ & & & \\
\hline BUN & 1.01 & $0.99-1.02$ & 0.46 & & & \\
\hline \multicolumn{7}{|l|}{ Perioperative variables } \\
\hline CPB time & 1.01 & $1.01-1.01$ & $<0.001$ & 1.01 & $1.00-1.01$ & $<0.001$ \\
\hline ACC time & 1.01 & $1.01-1.02$ & $<0.001$ & & & \\
\hline \multicolumn{7}{|l|}{ Transfusion } \\
\hline $\mathrm{RBC}$ & 1.48 & $1.38-1.59$ & $<0.001$ & & & \\
\hline PC & 1.24 & $1.19-1.30$ & $<0.001$ & & & \\
\hline FFP & 1.45 & $1.34-1.56$ & $<0.001$ & 1.22 & $1.10-1.34$ & $<0.001$ \\
\hline CRYO & 1.36 & $1.28-1.45$ & $<0.001$ & 1.16 & $1.07-1.26$ & $<0.001$ \\
\hline ECMO & 11.97 & $6.82-21.01$ & $<0.001$ & 4.11 & $1.95-8.63$ & $<0.001$ \\
\hline Re-exploration & 8.11 & $4.45-14.77$ & $<0.001$ & & & \\
\hline Hyperchloremia & 1.23 & $0.86-1.77$ & 0.25 & & & \\
\hline Total amount of NS & 1.07 & $0.93-1.23$ & 0.33 & & & \\
\hline $\mathrm{NS}(>1 \mathrm{~L})$ & 1.07 & $0.71-1.61$ & 0.76 & & & \\
\hline NS (>2 L) & 1.04 & $0.55-1.98$ & 0.91 & & & \\
\hline
\end{tabular}

RIFLE, Risk, Injury, Failure, Loss, End Stage; OR, odds ratio; Cl, confidence interval; CABG, coronary artery bypass graft; DM, diabetes mellitus; HTN, hypertension; CVA, cerebrovascular accident; LVEF, left ventricular ejection fraction; ARB, angiotensin receptor blockers; ACE, angiotensin-converting enzyme; GFR, Glomerular filtration rate using Cockcroft-Gault formula; BUN, blood urea nitrogen; CPB, cardiopulmonary bypass; ACC, aortic cross clamp; RBC, red blood cell; PC, platelet concentrate; FFP, fresh frozen plasma; CRYO, cryoprecipitate; ECMO, extracorporeal membrane oxygenation; NS, normal saline. 
Table S2 Risk factor analysis for renal replacement therapy

\begin{tabular}{|c|c|c|c|c|c|c|}
\hline \multirow{2}{*}{ Variables } & \multicolumn{3}{|c|}{ Univariate analysis } & \multicolumn{3}{|c|}{ Multivariable analysis } \\
\hline & OR & $95 \% \mathrm{Cl}$ & $P$ value & OR & $95 \% \mathrm{Cl}$ & $P$ value \\
\hline \multicolumn{7}{|l|}{ Preoperative variables } \\
\hline Sex (male) & 0.83 & $0.53-1.29$ & 0.41 & & & \\
\hline Age & 1.04 & $1.02-1.06$ & $<0.001$ & 1.03 & $1.00-1.05$ & 0.02 \\
\hline Type of operation & & & 0.02 & & & \\
\hline CABG & 1.88 & $0.86-4.10$ & 0.11 & & & \\
\hline Valve + CABG & 1.20 & $0.36-3.99$ & 0.76 & & & \\
\hline Aorta & 2.49 & $1.47-4.21$ & 0.00 & & & \\
\hline Others & 1.06 & $0.49-2.30$ & 0.87 & & & \\
\hline DM & 2.30 & $1.41-3.73$ & 0.00 & & & \\
\hline HTN & 1.43 & $0.92-2.21$ & 0.11 & & & \\
\hline CVA & 1.74 & $0.78-3.90$ & 0.18 & & & \\
\hline Euro score & 1.07 & $1.04-1.09$ & $<0.001$ & & & \\
\hline LVEF & 0.96 & $0.94-0.98$ & $<0.001$ & 0.97 & $0.95-0.99$ & 0.001 \\
\hline \multicolumn{7}{|l|}{ Medication } \\
\hline ARB & 1.51 & $0.97-2.35$ & 0.07 & & & \\
\hline Diuretics & 2.00 & $1.29-3.10$ & 0.00 & & & \\
\hline Hypoglycemic agents & 3.07 & $1.84-5.12$ & $<0.001$ & & & \\
\hline ACE inhibitor & 1.50 & $0.80-2.83$ & 0.20 & & & \\
\hline Insulin & 3.09 & $1.82-5.24$ & $<0.001$ & & & \\
\hline Statin & 1.31 & $0.84-2.05$ & 0.24 & & & \\
\hline Antiplatelet & 1.64 & $1.06-2.56$ & 0.03 & & & \\
\hline Hemoglobin & 0.70 & $0.62-0.78$ & $<0.001$ & 0.81 & $0.71-0.91$ & 0.001 \\
\hline Creatinine & 1.23 & $1.05-1.44$ & 0.01 & & & \\
\hline GFR & 0.98 & $0.97-0.98$ & $<0.001$ & 0.98 & $0.97-0.99$ & 0.006 \\
\hline Total bilirubin & 1.24 & $1.00-1.55$ & 0.05 & & & \\
\hline Albumin & 0.26 & $0.19-0.37$ & $<0.001$ & & & \\
\hline BUN & 1.06 & $1.04-1.08$ & $<0.001$ & & & \\
\hline \multicolumn{7}{|l|}{ Perioperative variables } \\
\hline CPB time & 1.01 & $1.01-1.01$ & $<0.001$ & 1.00 & $1.00-1.01$ & 0.008 \\
\hline ACC time & 1.01 & $1.01-1.02$ & $<0.001$ & & & \\
\hline \multicolumn{7}{|l|}{ Transfusion } \\
\hline $\mathrm{RBC}$ & 1.45 & $1.34-1.57$ & $<0.001$ & & & \\
\hline PC & 1.26 & $1.20-1.31$ & $<0.001$ & & & \\
\hline FFP & 1.43 & $1.32-1.55$ & $<0.001$ & & & \\
\hline CRYO & 1.45 & $1.34-1.56$ & $<0.001$ & 1.29 & $1.18-1.41$ & $<0.001$ \\
\hline ECMO & 17.71 & $9.74-32.22$ & $<0.001$ & 4.81 & $2.36-9.61$ & $<0.001$ \\
\hline Re-exploration & 10.53 & $5.47-20.29$ & $<0.001$ & & & \\
\hline Hyperchloremia & 1.12 & $0.69-1.82$ & 0.64 & & & \\
\hline Total amount of NS & 0.98 & $0.79-1.22$ & 0.89 & & & \\
\hline $\mathrm{NS}(>1 \mathrm{~L})$ & 1.25 & $0.74-2.12$ & 0.40 & & & \\
\hline NS (>2 L) & 0.85 & $0.34-2.15$ & 0.74 & & & \\
\hline
\end{tabular}

OR, odds ratio; $\mathrm{Cl}$, confidence interval; CABG, coronary artery bypass graft; DM, diabetes mellitus; HTN, hypertension; CVA, cerebrovascular accident; LVEF, left ventricular ejection fraction; ARB, angiotensin receptor blockers; ACE, angiotensin-converting enzyme; GFR, Glomerular filtration rate using Cockcroft-Gault formula; BUN, blood urea nitrogen; CPB, cardiopulmonary bypass; ACC, aortic cross clamp; RBC, red blood cell; PC, platelet concentrates; FFP, fresh frozen plasma; CRYO, cryoprecipitate; ECMO, extracorporeal membrane oxygenation; NS, normal saline. 
Table S3 Risk factor analysis for in-hospital mortality

\begin{tabular}{|c|c|c|c|c|c|c|}
\hline \multirow{2}{*}{ Variables } & \multicolumn{3}{|c|}{ Univariate analysis } & \multicolumn{3}{|c|}{ Multivariable analysis } \\
\hline & $\mathrm{HR}$ & $95 \% \mathrm{Cl}$ & $P$ value & $\mathrm{HR}$ & $95 \% \mathrm{Cl}$ & $\mathrm{P}$ value \\
\hline \multicolumn{7}{|l|}{ Preoperative variables } \\
\hline Sex (male) & 0.99 & $0.61-1.62$ & 0.97 & & & \\
\hline Age & 1.05 & $1.03-1.07$ & $<0.001$ & 1.05 & $1.02-1.07$ & $<0.001$ \\
\hline Type of operation & & & 0.10 & & & \\
\hline CABG & 1.83 & $0.77-4.38$ & 0.17 & & & \\
\hline Valve + CABG & 2.76 & $1.08-7.09$ & 0.03 & & & \\
\hline Aorta & 1.94 & $1.03-3.63$ & 0.04 & & & \\
\hline Others & 1.25 & $0.55-2.83$ & 0.59 & & & \\
\hline DM & 2.63 & $1.56-4.42$ & $<0.001$ & & & \\
\hline HTN & 1.64 & $1.01-2.69$ & 0.05 & & & \\
\hline CVA & 1.27 & $0.46-3.49$ & 0.64 & & & \\
\hline Euro score & 1.06 & $1.05-1.08$ & $<0.001$ & & & \\
\hline LVEF & 0.96 & $0.94-0.98$ & $<0.001$ & & & \\
\hline \multicolumn{7}{|l|}{ Medication } \\
\hline ARB & 1.48 & $0.90-2.42$ & 0.12 & & & \\
\hline Diuretics & 1.70 & $1.04-2.78$ & 0.03 & & & \\
\hline Hypoglycemic agents & 2.77 & $1.57-4.87$ & $<0.001$ & & & \\
\hline ACE inhibitor & 2.09 & $1.12-3.91$ & 0.02 & & & \\
\hline Insulin & 4.32 & $2.55-7.34$ & $<0.001$ & 2.06 & $1.18-3.61$ & 0.01 \\
\hline Statin & 1.23 & $0.74-2.04$ & 0.42 & & & \\
\hline Antiplatelet & 2.35 & $1.44-3.83$ & 0.001 & & & \\
\hline Hemoglobin & 0.60 & $0.53-0.68$ & $<0.001$ & 0.80 & $0.71-0.91$ & $<0.001$ \\
\hline Creatinine & 1.41 & $1.27-1.57$ & $<0.001$ & & & \\
\hline GFR & 0.97 & $0.96-0.98$ & $<0.001$ & & & \\
\hline Total bilirubin & 1.41 & $1.22-1.64$ & $<0.001$ & & & \\
\hline Albumin & 0.26 & $0.19-0.35$ & $<0.001$ & & & \\
\hline BUN & 1.04 & $1.03-1.06$ & $<0.001$ & & & \\
\hline \multicolumn{7}{|l|}{ Perioperative variables } \\
\hline CPB time & 1.01 & $1.01-1.01$ & $<0.001$ & 1.01 & $1.00-1.01$ & 0.002 \\
\hline ACC time & 1.01 & $1.01-1.01$ & $<0.001$ & & & \\
\hline \multicolumn{7}{|l|}{ Transfusion } \\
\hline $\mathrm{RBC}$ & 1.24 & $1.20-1.28$ & $<0.001$ & & & \\
\hline PC & 1.20 & $1.17-1.24$ & $<0.001$ & & & \\
\hline FFP & 1.23 & $1.20-1.27$ & $<0.001$ & 1.12 & $1.05-1.19$ & $<0.001$ \\
\hline CRYO & 1.31 & $1.23-1.39$ & $<0.001$ & & & \\
\hline ECMO & 39.88 & $24.34-65.33$ & $<0.001$ & 12.60 & $7.42-21.41$ & $<0.001$ \\
\hline Re-exploration & 14.75 & $8.37-26.00$ & $<0.001$ & & & \\
\hline Hyperchloremia & 0.86 & $0.48-1.53$ & 0.60 & & & \\
\hline Total amount of NS & 1.08 & $0.89-1.32$ & 0.45 & & & \\
\hline $\mathrm{NS}(>1 \mathrm{~L})$ & 1.20 & $0.67-2.18$ & 0.54 & & & \\
\hline NS (>2 L) & 1.17 & $0.47-2.90$ & 0.74 & & & \\
\hline
\end{tabular}

HR, hazard ratio; $\mathrm{Cl}$, confidence interval; CABG, coronary artery bypass graft; DM, diabetes mellitus; HTN, hypertension; CVA, cerebrovascular accident; LVEF, left ventricular ejection fraction; ARB, angiotensin receptor blockers; ACE, angiotensin-converting enzyme; GFR, Glomerular filtration rate using Cockcroft-Gault formula; BUN, blood urea nitrogen; CPB, cardiopulmonary bypass; ACC, aortic cross clamp; RBC, red blood cell; PC, platelet concentrates; FFP, fresh frozen plasma; CRYO, cryoprecipitate; ECMO, extracorporeal membrane oxygenation; NS, normal saline. 